\title{
Erratum to: Artificial neural networks and their potentialities in analyzing budget health data: an application for Italy of what-if theory
}

\author{
Paolo Massimo Buscema ${ }^{1,2} \cdot$ Guido Maurelli $^{1}$ • \\ Francesco Saverio Mennini ${ }^{3} \cdot$ Lara Gitto $^{3} \cdot$ Simone Russo $^{3,4}$. \\ Matteo Ruggeri ${ }^{5} \cdot$ Silvia Coretti $^{5} \cdot$ Americo Cicchetti $^{5}$
}

Published online: 28 June 2016

(C) Springer Science+Business Media Dordrecht 2016

\section{Erratum to: Qual Quant DOI 10.1007/s11135-016-0329-y}

In the original publication of the article, the Italian quotes starts from "La tecnica" and ends with "della conoscenza" under the heading "Methods" which has been included by mistake should be removed and replaced with the following English translation "The auto-encoder

The online version of the original article can be found under doi:10.1007/s11135-016-0329-y.

Paolo Massimo Buscema

m.buscema@semeion.it

Guido Maurelli

g.maurelli@semeion.it

Francesco Saverio Mennini

f.mennini@uniroma2.it

Lara Gitto

gitto@ceis.uniroma2.it

Simone Russo

simone.russo82@hotmail.it

Matteo Ruggeri

mruggeri@rm.unicatt.it

Silvia Coretti

silvia.coretti@rm.unicatt.it

Americo Cicchetti

acicchetti@rm.unicatt.it

1 SEMEION Research Centre of Sciences of Communication, Rome, Italy

2 Department of Mathematical and Statistical Sciences, University of Colorado, Denver, CO, USA

3 CEIS - Economic Evaluation and HTA (EEHTA), Faculty of Economics, University of Rome Tor Vergata, Rome, Italy

4 Department of Statistics, University of Rome La Sapienza, Rome, Italy

5 ALTEMS, Università Cattolica del Sacro Cuore, Rome, Italy 
technology allows the optimization of the learning process. The purpose of this type of learning (autoencoder) is to measure the ability to understand the logic of a dataset. It is, in other words, a sort of "calibration mechanism" of knowledge". 